
\title{
28 Research Suare \\ Risk Factors and Severity of Keratoconus on the East Coast of China
}

\section{Yujie Mou}

Eye Center, the Second Affiliated Hospital of Zhejiang University, School of Medicine

\section{Qiyu Qin}

Eye Center, the Second Affiliated Hospital of Zhejiang University, School of Medicine

\section{Xiaodan Huang}

Eye Center, the Second Affiliated Hospital of Zhejiang University, School of Medicine

\section{Xiuming Jin ( $\sim$ lzyjxm@zju.edu.cn )}

The Second Affiliated Hospital of Zhejiang University, School of Medicine

\section{Research Article}

Keywords: Keratoconus, Demography, Severity, Hydrops, China

Posted Date: September 28th, 2021

DOI: https://doi.org/10.21203/rs.3.rs-878694/v1

License: (c) (i) This work is licensed under a Creative Commons Attribution 4.0 International License. Read Full License

Version of Record: A version of this preprint was published at International Ophthalmology on January 17th, 2022. See the published version at https://doi.org/10.1007/s10792-022-02212-w. 


\section{Abstract}

Purpose: This study aims to investigate the characteristics, potential risk factors and associated severity of keratoconus on the east coast of China.

Methods: In this prospective case-control study, patients diagnosed with keratoconus were invited to fill in the online electronic questionnaire designed with the help of the App named WenJuanXing. Several questions like, sex, age of diagnosis and eye care habits were administered to each subject through this e-questionnaire. Corneal topographies at diagnosis taken from Pentacam were also collected.

Results: 391 Chinese keratoconus outpatients were enrolled in this study, among which 159 patients were newly diagnosed. Only 3 patients $(0.77 \%)$ had a positive family history of keratoconus. Atopy could be identified in $66.0 \%$ of our patients. A total of 357 patients $(91.3 \%)$ mentioned the habit of eye rubbing, with 191 patients $(48.8 \%)$ rubbing their eyes frequently. Patients with younger age at onset $(P=0.005)$, male sex $(P=0.042)$, frequent eye rubbing $(P=0.003)$ and smoke $(P=0.002)$ were found significantly associated with increased risk of acute hydrops. In newly diagnosed patients, $79.9 \%$ had the maximum keratometry (Kmax) more than 52D, with males and patients aged 21-30 being even more serious $(P=0.009 ; P=0.005)$. Besides, in patients aged over 21 , frequent eye rubbing was significantly associated with steeper Kmax and more severe astigmatism (all $\mathrm{P} \bowtie 0.05$ ).

Conclusion: Our results suggest that frequent eye rubbing is closely related to the severity of keratoconus in Chinese patients. Chinese keratoconus patients always had much serious disease condition at the time of diagnosis. Thus, increasing the publicity of keratoconus and advocating appropriate eye care habits are needed urgently in China.

\section{Introduction}

Keratoconus $(\mathrm{KC})$ is a functional and progressive corneal disorder which is characterized by progressive thinning and ectasia of central cornea ${ }^{1}$. KC patients generally start with initial symptoms of blurred vision, nearsightedness, irregular astigmatism and eventually result in acute corneal hydrops and irreversible loss of vision ${ }^{2,3}$. Although several exhilarating diagnostic and therapeutic methods have been enrolled in clinical application, aetiology and pathogenesis of $\mathrm{KC}$ still remain elusive ${ }^{4}$.

Previous studies have proposed that several genetic and environmental factors may have contribution to the development of $\mathrm{KC}^{5}$. Characteristics and risk factors of $\mathrm{KC}$ vary wildly in different regions and races. The estimated prevalence of $K C$ ranges from 0.3 to 3300 per 100,000 in different populations ${ }^{6-8}$. The research of 2012 Beijing Eye Study showed a prevalence of $0.9 \% \pm 0.2 \%$ among Chinese population aged over 50 years ${ }^{9}$. Based on such a huge population, the number of potential $\mathrm{KC}$ patients might be enormous in China. Articles have unveiled the relationship between $\mathrm{KC}$ associated genetic loci (VSX1, IL1A, IL1B, COL4A3 and COL4A4) and Chinese Han ethnicity ${ }^{10-12}$. However, epidemiological study of KC 
among Chinese population is kind of a blank, except a research from Singapore in which only $60.3 \%$ of their subjects were made up of Chinese in 2011 and a research that simply illustrated several clinical features of Chinese KC patients in $2005^{13,14}$. Consequently, confusions on the severity and epidemiological factors such as positive family history, eye rubbing and atopy among Chinese KC patients are still exist.

In this study, detailed epidemiological information of $391 \mathrm{KC}$ patients and relevant corneal parameters of 159 newly diagnosed patients were enrolled to investigate the clinical characteristics, risk factors and severity of KC on the east coast of China.

\section{Methods}

\section{Subjects}

Patients mainly came from regions on the east coast of China. Patients including the first-visit and returnvisit $\mathrm{KC}$ patients who met the $\mathrm{KC}$ diagnostic criterion were recruited from people who came directly to clinics. This prospective case-control study was conducted at the Eye Center of the Second Affiliated Hospital of Zhejiang University School of Medicine. The study followed the Declaration of Helsinki and was approved by Ethics Committee of the Second Affiliated Hospital of Zhejiang University School of Medicine. A written informed consent was obtained from each participant.

\section{Diagnosis of KC}

The diagnosis of the KC was made of using Pentacam (Pentacam; Oculus, Inc, Wetzlar, Germany) based on the Belin-Ambrósio enhanced ectasia display (BAD), which evaluated elevation data (anterior and posterior), pachymetry distribution and keratometry of the cornea. Typical symptoms (progressing vision loss and astigmatism) and signs evaluating by slit lamp microscope examinations (Fleischer's ring and Vogt' stripes) were also supplementary information. Potential subjects with non-keratoconus ocular disease such as corneal ulcers and trauma, keratitis, cataracts, intraocular lens implants, macular disease and severe systematic diseases were not eligible for enrollment.

\section{The online questionnaire}

All identified KC patients were invited to scan a Q-R code that we posted on the wall of consulting room and fulfilled the online questionnaire. We designed this self-administered e-questionnaire with the help of the App named WenJuanXing, a professional online questionnaire platform. Questions of demographics such as the onset age, gender, ethnicity and potential risk factors such as eye-care status, contact lens wear, family history of KC, eye rubbing and atopy were included.

\section{The analysis of cornea topography}

For corneal topography examination assessing by Pentacam, flat (K1) and steep (K2) keratometric readings, maximum simulated keratometry (Kmax), corneal thickness of the thinnest point of the cornea (thinnest pachymetry) and astigmatism were recorded. The stage of keratoconus was classified as mild 
when the greatest keratometric reading (Kmax) was less than $45 \mathrm{dpt}$, moderate when Kmax was between 45 and $52 \mathrm{dpt}$ and severe when Kmax was more than $52 \mathrm{dpt}$. Kmax has been typically considered to be a reliable quantitative clinical variable to assess the severity of Keratoconus ${ }^{15,16}$.

\section{Data analysis}

The epidemiology data obtained from e-questionnaire were described by number of patients while the data of cornea parameters and therapy were described by number of eyes. Statistical significance was set at $\mathrm{P}<0.05$ and data analysis were performed using SPSS (version 25, IBM, Armonk, NY, United States). Data were presented as means \pm SD. Mann-Whitney $U$ test was used for the comparison of continuous variables and $\chi^{2}$-test was used for counting data.

\section{Results}

\section{Demographic data}

As shown in Table 1, a total of 391 Chinese KC patients with 582 eyes were included in this research, including $289(73.9 \%)$ males and $102(26.1 \%)$ females. Of these, $159(40.7 \%)$ patients were newly diagnosed during the entire study process. The average age at the onset of symptoms was $20.22 \pm 6.16$ years (range from 6 to 49 ) and the average age of definite diagnosis was $22.24 \pm 6.18$ years (range from 7 to 50). 246 (62.9\%) patients had bilateral KC and unilateral KC was found in 145 (37.1\%) patients. The clinical symptoms and therapeutic managements of 582 eyes of 391 patients were shown in Table 2.

Table 1

Demographic characteristics of KC patients

\begin{tabular}{|ll|}
\hline & Patients with KC \\
\hline Male & $289(73.9 \%)$ \\
\hline Female & $102(26.1 \%)$ \\
\hline Age at the onset $(\mathrm{y})$ & $20.22 \pm 6.16(6-49)$ \\
\hline Age at the diagnosis $(\mathrm{y})$ & $22.24 \pm 6.18(7-50)$ \\
\hline Bilateral KC & $246(62.9 \%)$ \\
\hline
\end{tabular}


Table 2

Clinical symptoms and therapeutic managements of KC patients

Eyes with $\mathrm{KC}(n=582)$

Clinical symptoms

Refractive error uncorrected by glasses $\quad 244$

Poor visual acuity uncorrected by glasses 246

Image distortion $\quad 111$

Eye pain, tearing, sore and swollen $\quad 42$

Protrusion of cornea $\quad 37$

Corneal rupture, swelling $\quad 12$

Asymptomatic 26

Treatment

Without Treatment 41

Glasses Only $\quad 44$

Rigid gas permeable contact lens $\quad 242$

Corneal collagen crosslinking $\quad 213$

Keratoplasty 42

\section{Risk factors of KC in China}

Potential risk factors were demonstrated in Table 3. Only $3(0.77 \%)$ patients revealed a positive family history of KC. For ocular-related risk factors, the most common disease among our patients was allergic conjunctivitis of 194 (49.62\%) subjects, followed by high myopia of $47(12.02 \%)$ subjects and dry eye of $32(8.18 \%)$ subjects. For systematic diseases, 109 (27.88\%) patients had allergic rhinitis and $26(6.6 \%)$ patients had diabetes. Most of the KC patients (91.30\%) had a habit of eye rubbing and only $69(17.65 \%)$ patients had a habit of smoking. 
Table 3

Potential risk factors of $\mathrm{KC}$ patients

\begin{tabular}{|ll|}
\hline Exposure Factor & $\mathbf{N}(\%)$ \\
\hline Family history of KC & $3(0.77 \%)$ \\
\hline High myopia & $47(12.02 \%)$ \\
\hline Allergic conjunctivitis & $194(49.62 \%)$ \\
\hline Dry eye & $32(8.18 \%)$ \\
\hline Keratitis & $18(4.60 \%)$ \\
\hline Trichiasis & $10(2.56 \%)$ \\
\hline Diabetes & $26(6.65 \%)$ \\
\hline Allergic rhinitis & $109(27.88 \%)$ \\
\hline Eczema & $33(8.44 \%)$ \\
\hline Urticaria & $30(7.67 \%)$ \\
\hline Asthma & $22(5.63 \%)$ \\
\hline Smoking & $69(17.65 \%)$ \\
\hline Eye rubbing & $357(91.30 \%)$ \\
\hline
\end{tabular}

\section{The severity of $\mathrm{KC}$ related to age and gender}

159 corneal topographies of newly diagnosed eyes taken from Pentacam were collected. In bilateral patients, more severe eyes were chosen for further analysis. Mean flat (K1) and steep (K2) keratometry were to be $48.76 \pm 8.62$ and $52.65 \pm 9.45$ dioptres (D) respectively. Average maximum simulated keratometry (Kmax) was $60.57 \pm 12.79 \mathrm{D}$ and average astigmatism was $3.83 \pm 2.16$. Mean thinnest pachymetry was $447.55 \pm 48.19 \mathrm{um}$. When divided by ages, patients under 20 years old and aged $21-30$ had significantly steeper Kmax compared with patients aged over 31. Besides, compared with patients younger than 21, patients aged 21-30 had much thinner thinnest pachymetry (Table 4). When divided by gender, Kmax in male patients was significantly higher than that in female patients, while there were no differences in K1, K2, thinnest pachymetry and astigmatism between males and females (Table 5). 
Table 4

Comparison of cornea topography variables in different age groups

\begin{tabular}{|c|c|c|c|c|c|c|}
\hline & Age $<20$ & Age 21-30 & Age $>31$ & $\mathrm{P}_{1}$ & $\mathrm{P}_{2}$ & $P_{3}$ \\
\hline K1 & $48.81 \pm 6.74$ & $49.53 \pm 10.68$ & $46.01 \pm 4.26$ & 0.695 & 0.245 & 0.347 \\
\hline K2 & $52.92 \pm 7.15$ & $53.33 \pm 11.83$ & $49.61 \pm 4.84$ & 0.279 & 0.108 & 0.384 \\
\hline Kmax & $60.77 \pm 10.23$ & $61.95 \pm 15.47$ & $55.00 \pm 6.07$ & 0.841 & $0.02^{*}$ & $0.005^{*}$ \\
\hline $\begin{array}{l}\text { Thinnest } \\
\text { pachymetry }\end{array}$ & $\begin{array}{l}457.10 \pm \\
52.04\end{array}$ & $\begin{array}{l}437.50 \pm \\
47.70\end{array}$ & $\begin{array}{l}455.71 \pm \\
29.46\end{array}$ & $0.011^{*}$ & 0.507 & 0.149 \\
\hline Astigmatism & $4.16 \pm 2.18$ & $3.72 \pm 2.19$ & $3.26 \pm 1.90$ & 0.176 & 0.117 & 0.576 \\
\hline
\end{tabular}

Table 5

Comparison of cornea topography variables in different gender groups

\begin{tabular}{|llll|}
\hline & Male & Female & $P$ \\
\hline K1 & $48.83 \pm 7.83$ & $48.57 \pm 10.61$ & 0.394 \\
\hline K2 & $52.82 \pm 8.31$ & $52.19 \pm 12.16$ & 0.101 \\
\hline Kmax & $61.32 \pm 10.85$ & $58.73 \pm 16.64$ & $0.009^{*}$ \\
\hline Thinnest pachymetry & $444.38 \pm 48.85$ & $455.31 \pm 46.14$ & 0.242 \\
\hline Astigmatism & $3.95 \pm 2.06$ & $3.52 \pm 2.37$ & 0.163 \\
\hline * $p<0.05$ for significant difference & & \\
\hline
\end{tabular}

KC severity related to eye rubbing

In $391 \mathrm{KC}$ patients, $91.3 \%$ mentioned the habit of eye rubbing while only $8.7 \% \mathrm{KC}$ patients never rubbed their eyes. The analysis of the association between eye rubbing and Kmax or astigmatism in 159 newly diagnosed patients was shown in Table 6. Patients who frequently rubbed their eyes were found to have a significantly steeper Kmax and more severe astigmatism while no difference was found in patients aged under 20 (Table 6). No significant relationships were found between eye rubbing and K1, K2 and thinnest pachymetry (data not shown). 
Table 6

Effects of frequent eye rubbing on Kmax and astigmatism in different age groups

\begin{tabular}{|lllllll|}
\hline \multicolumn{2}{|c|}{ Kmax } & \multicolumn{5}{c|}{ Astigmatism } \\
\hline & Eye rubbing & $\begin{array}{l}\text { Without eye } \\
\text { rubbing }\end{array}$ & $p$ & $\begin{array}{l}\text { Eye } \\
\text { rubbing }\end{array}$ & $\begin{array}{l}\text { Without eye } \\
\text { rubbing }\end{array}$ & $p$ \\
\hline Age $<20$ & $\begin{array}{l}61.25 \pm \\
10.93\end{array}$ & $60.38 \pm 9.75$ & 0.91 & $3.96 \pm$ & $4.39 \pm 2.08$ & 0.377 \\
\hline Age $21-$ & $\begin{array}{l}64.01 \pm \\
\text { 30 }\end{array}$ & $60.19 \pm 16.69$ & $0.043^{*}$ & $4.25 \pm$ & $3.25 \pm 2.17$ & $0.032^{*}$ \\
\hline Age $>31$ & $57.38 \pm 6.25$ & $51.13 \pm 3.26$ & $0.01^{*}$ & $3.76 \pm$ & $2.50 \pm 1.56$ & $0.005^{*}$ \\
\hline${ }^{*} p<0.05$ for significant difference & & & & \\
\hline
\end{tabular}

\section{Risk factors for corneal hydrops}

Among 391 enrolled patients, 12 patients had corneal acute hydrops, one of the most severe complications of KC. By comparing patients who had hydrops with those did not, Patients with younger age at onset $(P=0.005)$, eye rubbing $(P=0.003)$, and habit of smoking $(P=0.002)$ were found to have an increased risk for developing corneal hydrops. Besides, male keratoconus patients were much easier to develop into corneal hydrops than female ones $(P=0.042)$. However, allergic diseases and alcoholic were found having no relationship with the occurrence of corneal hydrops (Table 7). 
Table 7

Risk factors for developing corneal hydrops in patients with KC

\begin{tabular}{|c|c|c|c|}
\hline Variables & No Hydrops & Hydrops & $\mathbf{P}$ \\
\hline Male sex, n (\%) & $277(70.84 \%)$ & $12(100.00 \%)$ & $0.042^{*}$ \\
\hline Bilateral, n (\%) & $238(62.80 \%)$ & $8(66.67 \%)$ & $0.785^{\star}$ \\
\hline Age at onset of symptoms & $20.55 \pm 6.28$ & $16.25 \pm 1.76$ & $0.005^{\Phi}$ \\
\hline Family history of keratoconus & $3(0.79 \%)$ & $0(0 \%)$ & $1^{*}$ \\
\hline History of allergic diseases & $250(66.0 \%)$ & $8(66.67 \%)$ & $0.960^{*}$ \\
\hline Alcoholic & $54(14.35 \%)$ & $4(33.33 \%)$ & $0.156^{\star}$ \\
\hline Smoke & $44(11.61 \%)$ & $5(41.67 \%)$ & $0.002^{\star}$ \\
\hline Contact lens use & $43(11.35 \%)$ & $0(0 \%)$ & $0.377^{*}$ \\
\hline Excessive use of eye & $265(70.0 \%)$ & 10(83.33\%) & $0.317^{\star}$ \\
\hline Frequent eye rubbing & $184(48.55 \%)$ & $11(91.7 \%)$ & $0.003^{*}$ \\
\hline Conjunctivitis & $188(49.61 \%)$ & $6(50.00 \%)$ & $0.978^{\star}$ \\
\hline \multicolumn{4}{|l|}{$\star$ Using $\chi^{2}$-test } \\
\hline \multicolumn{4}{|l|}{ * Using Fisher exact test } \\
\hline Ф Using Mann-Whitney test & & & \\
\hline
\end{tabular}

\section{Discussion}

Although $\mathrm{KC}$ has been well documented in Western populations and some Asian countries, seldom was the epidemiology study on $\mathrm{KC}$ done in Chinese populations. This study aimed to demonstrate the basic clinical profiles of KC in Chinese patients.

The mean age of diagnosis was $22.24 \pm 6.18$ years in our study, which is similar with most other Asian or Western studies (ranged from $20.00 \pm 6.4$ to $24.05 \pm 8.97$ ) ${ }^{17,18}$. Published male and female ratios of KC varied from 1:1.1 to 2.5:1 in other Asian countries and 1:2 to 3:2 in Western countries ${ }^{13}$. Our research showed a gender ratio of 2.83:1, which is slightly larger than other Asian researches. The skewed sex ratios with high male majority in China may account for this.

Three $(0.77 \%)$ patients revealed a positive family history of $\mathrm{KC}$ in our study, which is much lesser than the published rates in other countries that vary from $5-23.5 \%{ }^{19,20}$. The prospective research in Singapore showed $26.3 \%$ of their KC patients had asthma, while only $0.9 \%$ of patients had vasomotor rhinitis ${ }^{13}$. 
Instead of asthma, allergic conjunctivitis and allergic rhinitis were the most common diseases accompanying with Chinese KC patients we studied. And the incidence of atopy diseases among Chinese $\mathrm{KC}$ patients was $66.0 \%$, which is much higher than that cited by other studies ${ }^{17,21}$. Different populations, unknown hereditary and especially the environmental factors could be reasons for this. As the urbanization and industrialization in China is accompanied by bad air quality, and the prevalence of asthma in China has been increasing in recent years ${ }^{22}$, the impact of deteriorating environment on $\mathrm{KC}$ should not be underestimated.

The association between eye rubbing and KC has been validated for many years. In 2000, Bawazeer et al found that eye rubbing was the most significant risk factor for $\mathrm{KC}$ in their multivariate analysis ${ }^{23}$. In-depth studies have illuminated the underlying mechanisms between eye rubbing and $\mathrm{KC}$, as the microtrauma of epithelium caused by eye rubbing may cause abnormal improved inflammatory factors, such as IL- 6 and TNF-a, which may in turn contribute to apoptosis of keratocytes ${ }^{24}$. Thus, gradual corneal thinning is inevitable. In our research, more than $90 \%$ of Chinese patients rubbed their eyes. And $48.5 \%$ of patients mentioned the habit of frequent eye rubbing, similar to the $48.2 \%$ in the case series by CLEK study ${ }^{25}$. Naderan M. et al found that in their Iranian patients, the higher frequency of eye rubbing and positive family history were associated with a more severe clinical stage of $\mathrm{KC}^{26}$. Similarly, we found in Chinese patients aged over 21, frequent eye rubbing significantly aggravate the disease condition, with these patients having more serious Kmax and more terrible astigmatism at diagnosis than those who did not. However, no relationship was found between positive family history, allergic diseases and the severity of $\mathrm{KC}$ in our study. But the correlation analysis showed patients with atopy were more likely to rub their eyes frequently $(P=0.045)$. This is consistent with the conclusion of Bawazeer et al that atopy may contribute to $\mathrm{KC}$ most probably via eye rubbing provoked by itching. As allergic diseases and eye rubbing were quite common among our patients, while their KC family history was sparsely positive. We speculate that differences may exist in the pathogenesis of $\mathrm{KC}$ between China and foreign countries, with environmental factors contributing more among Chinese patients than genetic ones.

$\mathrm{KC}$ was typically considered as a progressive disease that usually stabilizes by the third or fourth decade of life ${ }^{27}$, our data suggested that Chinese patients aged 21-30 have steeper Kmax and thinner thinnest pachymetry compared with other age group also accord with this. And similar with studies performed by other races ${ }^{28}$, the severity of Chinese $\mathrm{KC}$ was more serious in male patients than females. According to the value of Kmax, the majority of our patients $(79.9 \%)$ being diagnosed were in severe stage ( $(52 \mathrm{D})$. The proportion of patients with severe $\mathrm{KC}$ in our study was significantly larger than that published by other countries $^{4,25}$. We suppose the weak awareness of $\mathrm{KC}$ may lead to the serious condition of disease, as the majority of our patients never heard of $\mathrm{KC}$ and realized eye rubbing was an important risk factor for $\mathrm{KC}$ before diagnosis.

Similar with the research of Reena ${ }^{17}$, our study found patients with earlier age at onset and eye rubbing had an increased risk for corneal acute hydrops. Besides, we also found male patients with smoking habit were more likely to get hydrops. This may partly contradict with the study of Spoerl, which indicated

Page $10 / 14$ 
that fewer were smokers in $\mathrm{KC}$ patients received CXL and postulated that by-products of cigarette may lead to cross-linking of collagen, thus prevent the progression of $\mathrm{KC}^{29}$. Since the methodologies of these two studies were very different and no research had unveiled the inherent mechanism between smoke and KC, the interpretation should be with caution. Since all patients with history of smoking revealed the habit of frequent eye rubbing, we assume the behaviour of smoking may be related to terrible emotion that would cause vigorous eye rubbing and further lead to the happen of corneal hydrops.

Despite positive outcomes, there are still some limitations of our study: First, this study was conducted in a 3A (Class Three/Grade A) hospitals in China, Hangzhou. Thus, compared with general population, the patients reported in our study may have more serious disease. Second, as the majority of our patients reside in ZheJiang province, our results may have geographical limitations and may not reflect the accurate profile of all Chinese KC patients. Overall, further research awaits a more detailed study with evaluation of a larger number of Chinese cases.

\section{Conclusions}

Chinese KC patients that we investigated were always with serious disease at the time of first-visit, had a high prevalence of eye rubbing and atopy, but with little positive family history. And we found Chinese KC patients with younger age at onset, male sex, history of frequent eye rubbing and smoke habit were more easily to develop into acute hydrops, the deep understanding of such risk factors may help clinicians make more informed management strategies to delay or prevent the progression of this disease among Chinese patients. Besides, our data also showed frequent eye rubbing can significantly aggravate the disease in Chinese patients aged over 21, indicating the essential role of eye rubbing in the late pathogenesis of KC among Chinese patients. Thus, strengthening public health outreach of KC and indoctrinating appropriate eye care habits among Chinese populations are urgent affairs.

\section{Declarations}

Registration: The research was retrospectively registered and the trial registration number is ChiCTR1900021273 and the registration date is 2019/02/09.

Fundings: This work was supported by the Major Science and Technology Projects of Zhejiang Province (grant number 2017C03046) and (grant number 2020C03035) and the National Natural Science foundation of China (No. 81870624).

Conflict of interests: The authors declare they have no financial conflict of interest related to the study.

Data availability: The raw data used to support the findings of this study are available from the corresponding author upon request.

Author contributions: $\mathrm{YM}$ and $\mathrm{QQ}$ were responsible for experimental design, $\mathrm{HX}$ and $\mathrm{QQ}$ were responsible for data collection and analysis, YM and $\mathrm{XH}$ were responsible for article drafting and revising. 
Ethic approval: The study followed the Declaration of Helsinki and was approved by Ethics Committee of the Second Affiliated Hospital of Zhejiang University School of Medicine.

\section{References}

1. Ferdi AC, Nguyen V, Gore DM, Allan BD, Rozena JJ, Watson SL (2019) Keratoconus Natural Progression: A Systematic Review and Meta-analysis of 11,529 eyes. Ophthalmology 126(7):935945. doi:10.1016/j.ophtha.2019.02.029

2. Tatematsu-Ogawa Y, Yamada M, Kawashima M, Yamazaki Y, Bryce T, Tsubota K. The disease burden of keratoconus in patients' lives: comparisons to a Japanese normative sample(2008) Eye \& contact lens 34(1):13-6. doi:10.1097/ICL.0b013e3180515282

3. Kymes SM, Walline JJ, Zadnik K, Gordon MO. Quality of life in keratoconus(2004) American journal of ophthalmology 138(4):527-35. doi:10.1016/j.ajo.2004.04.031

4. Kok YO, Tan GF, Loon SC (2012) Review: keratoconus in Asia. Cornea 31(5):581-593. doi:10.1097/ICO.0b013e31820cd61d

5. Rabinowitz YS (2003) The genetics of keratoconus. Ophthalmology clinics of North America 16(4):607-620, vii

6. Hashemi H, Khabazkhoob M, Yazdani N, Ostadimoghaddam H, Norouzirad R, Amanzadeh K et al (2014) The prevalence of keratoconus in a young population in Mashhad, Iran. Ophthalmic physiological optics: the journal of the British College of Ophthalmic Opticians (Optometrists) 34(5):519-527. doi:10.1111/opo.12147

7. Gayton JL(2009) Etiology, prevalence, and treatment of dry eye disease. Clinical ophthalmology. 3:405 - 12. 10.2147/opth.s5555

8. Gokhale NS (2013) Epidemiology of keratoconus. Indian J Ophthalmol 61(8):382-383. doi:10.4103/0301-4738.116054

9. Xu L, Wang YX, Guo Y, You QS, Jonas JB (2012) Prevalence and associations of steep cornea/keratoconus in Greater Beijing. The Beijing Eye Study. PloS one 7(7):e39313. doi:10.1371/journal.pone.0039313

10. Guan T, Wang X, Zheng LB, Wu HJ, Yao YF (2017) Analysis of the VSX1 gene in sporadic keratoconus patients from China. BMC Ophthalmol 17(1):173. doi:10.1186/s12886-017-0567-3

11. Wang Y, Jin T, Zhang X, Wei W, Cui Y, Geng T et al (2013) Common single nucleotide polymorphisms and keratoconus in the Han Chinese population. Ophthalmic Genet 34(3):160-166. doi:10.3109/13816810.2012.743569

12. Wang Y, Wei W, Zhang C, Zhang X, Liu M, Zhu X et al (2016) Association of Interleukin-1 Gene Single Nucleotide Polymorphisms with Keratoconus in Chinese Han Population. Curr Eye Res 41(5):630635. doi:10.3109/02713683.2015.1045083

13. Khor WB, Wei RH, Lim L, Chan CM, Tan DT (2011) Keratoconus in Asians: demographics, clinical characteristics and visual function in a hospital-based population. Clin Exp Ophthalmol 39(4):299- 
307. doi:10.1111/j.1442-9071.2010.02458.x

14. Li SW, Li ZX, Shi WY, Zeng QY, Jin XM (2005) [Clinical features of 233 cases of keratoconus]. [Zhonghua yan ke za zhi]. Chinese journal of ophthalmology 41(7):610-613

15. Jun AS, Cope L, Speck C, Feng X, Lee S, Meng H et al (2011) Subnormal cytokine profile in the tear fluid of keratoconus patients. PloS one 6(1):e16437. doi:10.1371/journal.pone.0016437

16. Lema I, Duran JA, Ruiz C, Diez-Feijoo E, Acera A, Merayo J (2008) Inflammatory response to contact lenses in patients with keratoconus compared with myopic subjects. Cornea 27(7):758-763. doi:10.1097/ICO.0b013e31816a3591

17. Sharma R, Titiyal JS, Prakash G, Sharma N, Tandon R, Vajpayee RB (2009) Clinical profile and risk factors for keratoplasty and development of hydrops in north Indian patients with keratoconus. Cornea 28(4):367-370. doi:10.1097/IC0.0b013e31818cd077

18. Weed KH, MacEwen CJ, McGhee CN (2006) The variable expression of keratoconus within monozygotic twins: dundee University Scottish Keratoconus Study (DUSKS). Contact lens \& anterior eye:. the journal of the British Contact Lens Association 29(3):123-126. doi:10.1016/j.clae.2006.03.003

19. Owens H, Gamble G (2003) A profile of keratoconus in New Zealand. Cornea 22(2):122-125

20. Weed KH, MacEwen CJ, Giles T, Low J, McGhee CN (2008) The Dundee University Scottish Keratoconus study: demographics, corneal signs, associated diseases, and eye rubbing. Eye 22(4):534-541. doi:10.1038/sj.eye.6702692

21. Cozma I, Atherley C, James NJ(2005) Influence of ethnic origin on the incidence of keratoconus and associated atopic disease in Asian and white patients. Eye (London, England) 19(8):924-5; author reply 5-6. doi:10.1038/sj.eye.6701677

22. Chen F, Lin Z, Chen R, Norback D, Liu C, Kan H et al (2018) The effects of PM2.5 on asthmatic and allergic diseases or symptoms in preschool children of six Chinese cities, based on China, Children, Homes and Health (CCHH) project. Environmental pollution (Barking, Essex: 1987) 232:329 - 37. doi:10.1016/j.envpol.2017.08.072

23. Bawazeer AM, Hodge WG, Lorimer B (2000) Atopy and keratoconus: a multivariate analysis. Br J Ophthalmol 84(8):834-836

24. Galvis V, Sherwin T, Tello A, Merayo J, Barrera R, Acera A (2015) Keratoconus: an inflammatory disorder? Eye. (London England) 29(7):843-859. doi:10.1038/eye.2015.63

25. Zadnik K, Barr JT, Edrington TB, Everett DF, Jameson M, McMahon TT et al (1998) Baseline findings in the Collaborative Longitudinal Evaluation of Keratoconus (CLEK) Study. Investig Ophthalmol Vis Sci 39(13):2537-2546

26. Naderan M, Shoar S, Rezagholizadeh F, Zolfaghari M, Naderan M (2015) Characteristics and associations of keratoconus patients. Contact lens anterior eye: the journal of the British Contact Lens Association 38(3):199-205. doi:10.1016/j.clae.2015.01.008

27. Ertan A, Muftuoglu O (2008) Keratoconus clinical findings according to different age and gender groups. Cornea 27(10):1109-1113. doi:10.1097/IC0.0b013e31817f815a 
28. Gordon-Shaag A, Millodot M, Shneor E, Liu Y (2015) The genetic and environmental factors for keratoconus. BioMed research international 2015:795738. doi:10.1155/2015/795738

29. Spoerl E, Raiskup-Wolf F, Kuhlisch E, Pillunat LE(2008) Cigarette smoking is negatively associated with keratoconus. Journal of refractive surgery (Thorofare, NJ: 1995) 24(7):S737-40 